\title{
System Functions with Minimum Time-Bandwidth Product Based on the Higher Order Moments
}

\author{
Hrvoje Babic and Mladen Vucic \\ Faculty of Electrical Engineering and Computing \\ Unska 3, Zagreb, HR10000, Croatia \\ E-mail:nrvoje.babic@fer.hr,mladen.vucic@fer.hr
}

\begin{abstract}
Systems of finite order with minimum time-bandwidth products are considered. The time and frequency response spreads are defined by moments of the higher order in both domains. Minimizing the product of the moments, causal systems with the largest energy concentration in the time and frequency domain are obtained. The optimization is carried out for transfer functions up to the eighth order with two and four complex zeros. The moment orders are chosen from two to eight. The influence of the zeros and the moment order to system properties are considered. The complete data suitable for filter design are given.
\end{abstract}

\section{Introduction}

In many applications, the systems with small time spread of the impulse response for a given bandwidth are required [1]. As short signals require wide bandwidths, the optimum is a compromise given by minimum of the time and frequency product

$$
P_{n}=\alpha_{n} \beta_{n} .
$$

For the time spread $\alpha_{n}$ and frequency spread $\beta_{n}$ the various measures might be used [2]. The moments

$$
\begin{aligned}
& \alpha_{n}^{\mathrm{n}}=\frac{\int_{-\infty}^{\infty}\left(\mathrm{t}-\mathrm{t}_{\mathrm{m}}\right)^{\mathrm{n}} \mathrm{h}^{2}(\mathrm{t}) \mathrm{dt}}{\int_{-\infty}^{\infty} \mathrm{h}^{2}(\mathrm{t}) \mathrm{dt}}, \mathrm{n}=2,4, \ldots \\
& \beta_{\mathrm{n}}^{\mathrm{n}}=\frac{\frac{1}{2 \pi} \int_{-\infty}^{\infty} \omega^{\mathrm{n}}|\mathrm{H}(\omega)|^{2} \mathrm{~d} \omega}{\frac{1}{2 \pi} \int_{-\infty}^{\infty}|\mathrm{H}(\omega)|^{2} \mathrm{~d} \omega}, \mathrm{n}=2,4, \ldots
\end{aligned}
$$

enable a simple mathematical treatment and optimization.
The classic uncertainty principle is defined by the second moments. For noncausal signals the product satisfy expression [2]

$$
\mathrm{P}_{2} \geq \frac{1}{2} .
$$

The equality value is obtained for Gauss function. For causal signals, a similar relation and optimal functions have been found [3]. The finite order systems will only approximate the mentioned optimal functions obtained on the basis of the second moment, but the product will come very close to the theoretical limit $P_{2}=1 / 2$. The higher moments could give an additional insight in the systems with minimum time-frequency products and therefore generalize the mentioned problem. Furthermore, the real systems of the finite order typically have ringing. The general requirement is to make ringing small and short. In the optimization procedure, all these aspect should be present in the goal function. The use of integral criteria (2) and (3) is proposed because $\left(\mathrm{t}-\mathrm{t}_{\mathrm{m}}\right)^{\mathrm{n}}$ and $\omega^{\mathrm{n}}$ as weighting functions, will shorten the tails of the time and frequency responses. Such systems could be used for filters, signal generation, delay and pulse-forming networks. We have previously considered systems with various moments order in the time domain, while the frequency domain spread was given by the zeroth [4] or the second moment order [5].

\section{Moments and transfer functions}

Time spread and bandwidth definitions (2) and (3), are suitable for causal functions with corresponding change of integral limits. Thus, we define a measure of the impulse response spread by the n-th order central moment, and the bandwidth by the $\mathrm{n}$-th order moment, both normalized to the impulse response energy.

For optimization procedure in the complex domain, the criterion (1) could be expressed by the transfer function poles $p_{i}$, and zeros, $z_{i}$. The system function of the N-th order, with $\mathrm{M}$ zeros is given by 


$$
H(s)=H_{0} \frac{\prod_{i=1}^{M}\left(s-z_{i}\right)}{\prod_{j=1}^{N}\left(s-p_{j}\right)} .
$$

If the poles are simple, and $\mathrm{M}<\mathrm{N}$, the impulse response is

$$
h(t)=\sum_{r=1}^{N} K_{r} e^{p_{r} t}, \quad K_{r}=H_{0} \frac{\prod_{\substack{i=1 \\ j=1}}^{M}\left(p_{r}-z_{i}\right)}{\prod_{\substack{j \neq 1 \\ j \neq r}}^{N}\left(p_{r}-p_{j}\right)},
$$

where the pole residues are $K_{r}, r=1,2, \ldots, N$. The $n$-th moment of the impulse response can be expressed as function of poles, zeros and residues as

$$
\mathrm{m}_{\mathrm{n}}=(-1)^{\mathrm{n}+1} \sum_{\mathrm{i}=1}^{\mathrm{N}} \sum_{\mathrm{j}=1}^{\mathrm{N}} \mathrm{K}_{\mathrm{i}} \mathrm{K}_{\mathrm{j}} \sum_{\mathrm{k}=0}^{\mathrm{n}} \frac{\mathrm{n} !}{\mathrm{k} !} \frac{\mathrm{t}_{\mathrm{m}}^{\mathrm{k}}}{\left(\mathrm{p}_{\mathrm{i}}+\mathrm{p}_{\mathrm{j}}\right)^{\mathrm{n}-\mathrm{k}+1}} .
$$

Impulse response energy, which is in fact the zeroth order moment, is obtained from (7) for $\mathrm{n}=0$.

The n-th moment of the frequency response can be expressed by the impulse response derivative, using Parseval's relation

$$
\frac{1}{2 \pi} \int_{-\infty}^{\infty} \omega^{\mathrm{n}}|\mathrm{H}(\omega)|^{2} \mathrm{~d} \omega=\int_{-\infty}^{\infty}\left[\mathrm{h}^{(\mathrm{n} / 2)}(\mathrm{t})\right]^{2} \mathrm{dt} .
$$

Expression (8) might be also determined from (7) using Laplace transform of the derivative

$$
\mathrm{L}\left\{\mathrm{h}^{(\mathrm{n} / 2)}(\mathrm{t})\right\}=\mathrm{s}^{\mathrm{n} / 2} \mathrm{H}(\mathrm{s}), \mathrm{n}=2,4,6,8
$$

where residues $\mathrm{Q}_{\mathrm{r}}$ of the transform (9) are given by

$$
\begin{gathered}
\mathrm{h}^{(\mathrm{n} / 2)}(\mathrm{t})=\sum_{\mathrm{r}=1}^{\mathrm{N}} \mathrm{Q}_{\mathrm{r}} \mathrm{e}^{\mathrm{p}_{\mathrm{r}} \mathrm{t}}, \\
\mathrm{Q}_{\mathrm{r}}=\mathrm{H}_{0} \frac{\mathrm{p}_{\mathrm{r}}^{\mathrm{n} / 2} \prod_{\substack{\mathrm{i}=1 \\
\mathrm{j}}}^{\mathrm{M}}\left(\mathrm{p}_{\mathrm{r}}-\mathrm{z}_{\mathrm{i}}\right)}{\prod_{\substack{\mathrm{j} \neq \mathrm{r} \\
\mathrm{N}}}\left(\mathrm{p}_{\mathrm{r}}-\mathrm{p}_{\mathrm{j}}\right)},
\end{gathered}
$$

To ensure convergence of the moment integral (3), the integrand should decay as $1 / \omega^{2}$ or faster. Thus, the number of poles and zeros should satisfy inequality

$$
2 \mathrm{~N}-(2 \mathrm{M}+\mathrm{n}) \geq 2 \text { or } \mathrm{N} \geq \mathrm{M}+1+\mathrm{n} / 2
$$

\section{OPTIMIZATION PROCEDURE AND RESULTS}

Pole and zero positions of causal filters with minimum time-bandwidth product can be found by solving the problem

$$
\min _{z_{i}, p_{j}} P_{n}\left[z_{i}, p_{j}\right], i=1, . ., M \text { and } j=1, \ldots, N .
$$

It is more practical to use goal function with real variables. Therefore, the complex poles and zeros in (12) were separated into their real and imaginary parts; $\mathrm{z}_{\mathrm{i}}=\mathrm{z}_{\alpha \mathrm{i}}+\mathrm{i} \cdot \mathrm{z}_{\beta \mathrm{i}}$ and $\mathrm{p}_{\mathrm{j}}=\mathrm{p}_{\alpha \mathrm{j}}+\mathrm{i} \cdot \mathrm{p}_{\beta \mathrm{j}}$. Using this notation, the optimization problem can be formulated as

$$
\min _{z_{\alpha i}, z_{\beta i}, p_{\alpha j}, p_{\beta j}} P_{n}\left[z_{\alpha i}, z_{\beta i}, p_{\alpha j}, p_{\beta j}\right],
$$

$$
\begin{gathered}
i=1, . ., M / 2 \text { and } j=1, \ldots, N / 2 . \\
\min _{z_{\alpha i}, z_{\beta i}, p_{\alpha j}, p_{\beta j}, p_{\alpha N}} P_{n}\left[z_{\alpha i}, z_{\beta i}, p_{\alpha j}, p_{\beta j}, p_{\alpha N}\right], \\
i=1, . ., M / 2 \text { and } j=1, \ldots,(N-1) / 2 .
\end{gathered}
$$

for even and odd system orders, respectively. The complex poles and zeros of real systems come in conjugate pairs. Therefore, the indexes $i$ and $j$ in (13) and (14) are running to $M / 2$ and $N / 2$ only.

For searching minimum Quasi-Newton method with BFGS formula for Hessian matrix update [6] was used. Analytic expressions for gradients were used in order to avoid numerical errors that finite difference approximation might have caused. Gradient was calculated using forward mode of automatic differentiation, as it can be found for example in [7].

To get causal filters with minimum time-bandwidth product, the optimization is carried out for systems up to the eighth order, with one pair of complex zeros. The moment orders, $n=2,4,6$ and 8 were chosen. From (2) one can expect that optimization will force impulse response to concentrate around $t_{\mathrm{m}}$ and practically extend to $2 \mathrm{t}_{\mathrm{m}}$. The parameter $\mathrm{t}_{\mathrm{m}}$ is chosen to be 1 . This will not change the generality of the solution.

The numerical values of the zeros and poles normalized to $t_{m}=1$ are given in Tables I - IV, together with cutoff frequency $\omega_{3 \mathrm{~dB}}$. In all cases, poles are very nearly placed on the ellipses with ellipses center located in the complex plane origin, Figure 1.

\subsection{System based on the fourth order moment}

To illustrate behavior of the considered class of systems, the complete diagrams are given for the system 
Table I. Pole-zero parameters for the systems based on the second order moment, $t_{m}=1$.

\begin{tabular}{|c|c|c|c|}
\hline $\mathbf{N}$ & $\mathbf{p}_{\mathbf{j}}$ & $\mathbf{z}_{\mathbf{i}}$ & $\omega_{\mathbf{3 d B}}$ \\
\hline 4 & $-1.6419 \pm 3.6500 \mathrm{i}$ & $1.6862 \pm 8.6033 \mathrm{i}$ & 2.0960 \\
& $-1.9188 \pm 1.1834 \mathrm{i}$ & & \\
\hline \multirow{3}{*}{5} & $-1.9299 \pm 5.1496 \mathrm{i}$ & $2.1762 \pm 8.1422 \mathrm{i}$ & \\
& $-2.2893 \pm 2.4785 \mathrm{i}$ & & 2.3578 \\
\hline \multirow{6}{*}{6} & -2.3798 & & \\
& $-2.1233 \pm 6.6307 \mathrm{i}$ & $2.7984 \pm 8.8747 \mathrm{i}$ & \\
& $-2.5549 \pm 3.8224 \mathrm{i}$ & & 2.6245 \\
& $-2.7151 \pm 1.2540 \mathrm{i}$ & & \\
7 & $-2.2477 \pm 8.1113 \mathrm{i}$ & $3.4840 \pm 9.7917 \mathrm{i}$ & \\
& $-2.7506 \pm 5.2033 \mathrm{i}$ & & 2.8873 \\
& $-2.9723 \pm 2.5545 \mathrm{i}$ & & \\
\hline & -3.0385 & & \\
8 & $-2.3188 \pm 9.5898 \mathrm{i}$ & $4.2104 \pm 10.7574 \mathrm{i}$ & \\
& $-2.8929 \pm 6.6100 \mathrm{i}$ & & 3.1449 \\
& $-3.1714 \pm 3.8912 \mathrm{i}$ & & \\
\hline
\end{tabular}

Table II. Pole-zero parameters for the systems based on the fourth order moment, $t_{m}=1$.

\begin{tabular}{|c|c|c|c|}
\hline $\mathbf{N}$ & $\mathbf{p}_{\mathbf{j}}$ & $\mathbf{z}_{\mathbf{i}}$ & $\omega_{\mathbf{3 d B}}$ \\
\hline \multirow{3}{*}{5} & $-1.8803 \pm 4.5023 \mathrm{i}$ & $0.1312 \pm 10.9828 \mathrm{i}$ & \\
& $-2.3307 \pm 2.3016 \mathrm{i}$ & & 2.4026 \\
& -2.4799 & & \\
\hline & $-1.8844 \pm 5.5848 \mathrm{i}$ & $-0.3729 \pm 8.4803 \mathrm{i}$ & \\
6 & $-2.4503 \pm 3.4328 \mathrm{i}$ & & 2.5017 \\
& $-2.7212 \pm 1.1618 \mathrm{i}$ & & \\
\hline & $-1.8481 \pm 6.8160 \mathrm{i}$ & $-0.2756 \pm 8.1069 \mathrm{i}$ & \\
7 & $-2.5231 \pm 4.6230 \mathrm{i}$ & & 2.6498 \\
& $-2.8829 \pm 2.3465 \mathrm{i}$ & & \\
& -3.0075 & & \\
& $-1.8432 \pm 8.1790 \mathrm{i}$ & $-0.1493 \pm 8.4291 \mathrm{i}$ & \\
8 & $-2.5835 \pm 5.8562 \mathrm{i}$ & & 2.8311 \\
& $-3.0105 \pm 3.5542 \mathrm{i}$ & & \\
\hline
\end{tabular}

with minimum product of fourth order moments in the time and the frequency domain. Impulse response is shown in Figure 2. It is quasi Gaussian response, with small time spread and undershoots less than $1.5 \%$. Step response overshoots are bellow $0.39 \%$, as can be seen from Figure 3. The product of rise-time $t_{\mathrm{r}}(10 \%$ to $90 \%)$ and bandwidth $\mathrm{f}_{3 \mathrm{~dB}}$ is approximately 0.348 .

Amplitude and group delay responses are shown Figure 4 and Figure 5, respectively, in a form suitable for comparison with classic filter approximations, given for example in [8]. The amplitude response is also quasi Gaussian. The group delay curves illustrate an
Table III. Pole-zero parameters for the systems based on the sixth order moment, $t_{m}=1$.

\begin{tabular}{|c|c|c|c|}
\hline $\mathbf{N}$ & $\mathbf{p}_{\mathbf{j}}$ & $\mathbf{z}_{\mathbf{i}}$ & $\omega_{3 \mathbf{d B}}$ \\
\hline & $-2.2591 \pm 5.4921 \mathrm{i}$ & $-0.7169 \pm 12.3673 \mathrm{i}$ & \\
6 & $-2.7620 \pm 3.3797 \mathrm{i}$ & & 2.6180 \\
& $-3.0063 \pm 1.1402 \mathrm{i}$ & & \\
\hline & $-2.1734 \pm 6.6284 \mathrm{i}$ & $-0.4266 \pm 8.8679 \mathrm{i}$ & \\
7 & $-2.7970 \pm 4.5410 \mathrm{i}$ & & 2.6788 \\
& $-3.1478 \pm 2.3061 \mathrm{i}$ & & \\
& -3.2628 & & \\
\hline & $-2.1471 \pm 7.9236 \mathrm{i}$ & $-0.1990 \pm 8.6568 \mathrm{i}$ & \\
8 & $-2.8461 \pm 5.7602 \mathrm{i}$ & & 2.8299 \\
& $-3.2743 \pm 3.5064 \mathrm{i}$ & & \\
\hline & $-3.4840 \pm 1.1777 \mathrm{i}$ & & \\
\hline
\end{tabular}

Table IV. Pole-zero parameters for the systems based on the eighth order moment, $t_{m}=1$.

\begin{tabular}{|c|c|c|c|}
\hline $\mathbf{N}$ & $\mathbf{p}_{\mathbf{j}}$ & $\mathbf{z}_{\mathbf{i}}$ & $\omega_{\mathbf{3 d B}}$ \\
\hline & $-2.7084 \pm 6.5319 \mathrm{i}$ & $-1.0107 \pm 14.0961 \mathrm{i}$ & \\
7 & $-3.2355 \pm 4.4562 \mathrm{i}$ & & 2.8272 \\
& $-3.5413 \pm 2.2570 \mathrm{i}$ & & \\
& -3.6415 & & \\
& $-2.6013 \pm 7.7077 \mathrm{i}$ & $-0.4114 \pm 10.0460 \mathrm{i}$ & \\
8 & $-3.2362 \pm 5.6387 \mathrm{i}$ & & 2.8854 \\
& $-3.6333 \pm 3.4335 \mathrm{i}$ & & \\
\hline
\end{tabular}
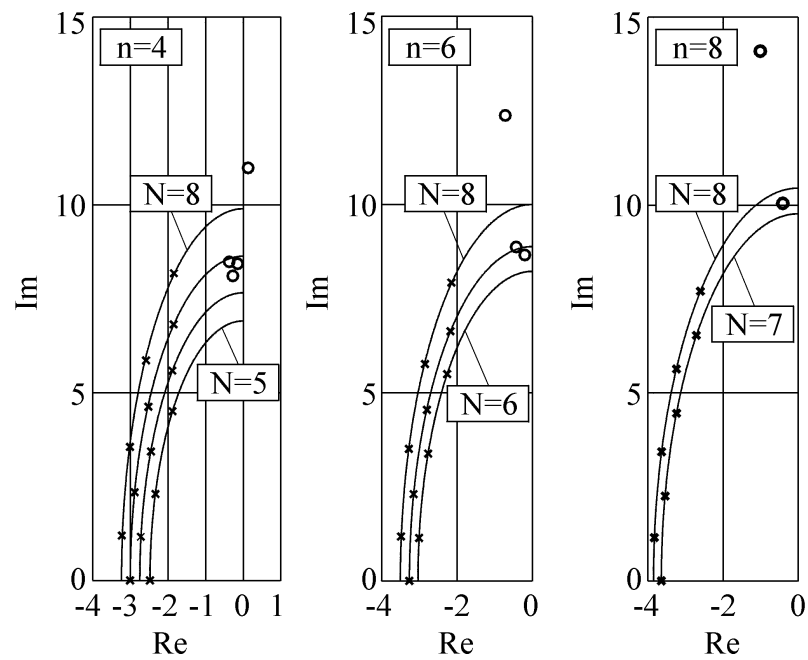

Figure 1. Pole-zero positions of the optimum systems based on the fourth, sixth and eighth order moment, normalized to $t_{m}=1$. 


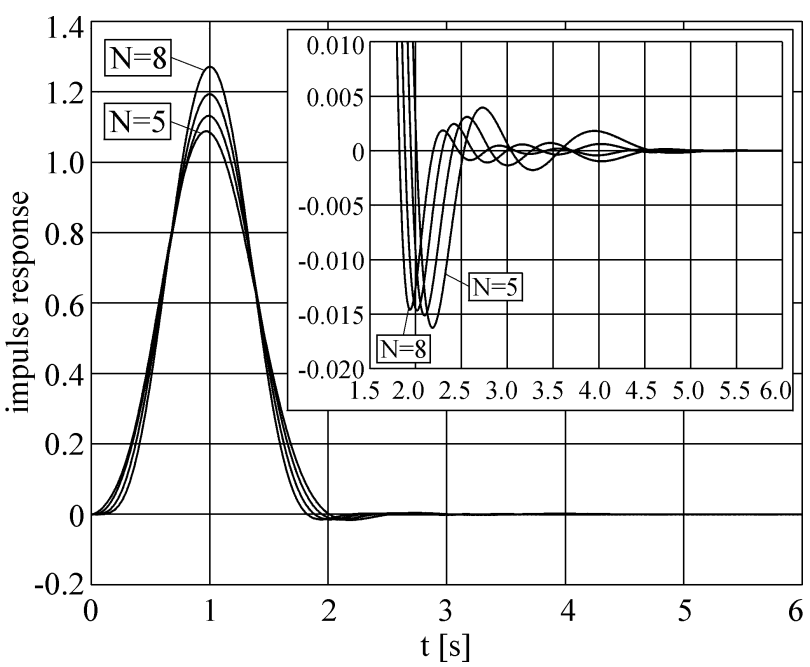

Figure 2. Impulse response of the optimum systems based on the fourth order moment, normalized to $t_{m}=1$.

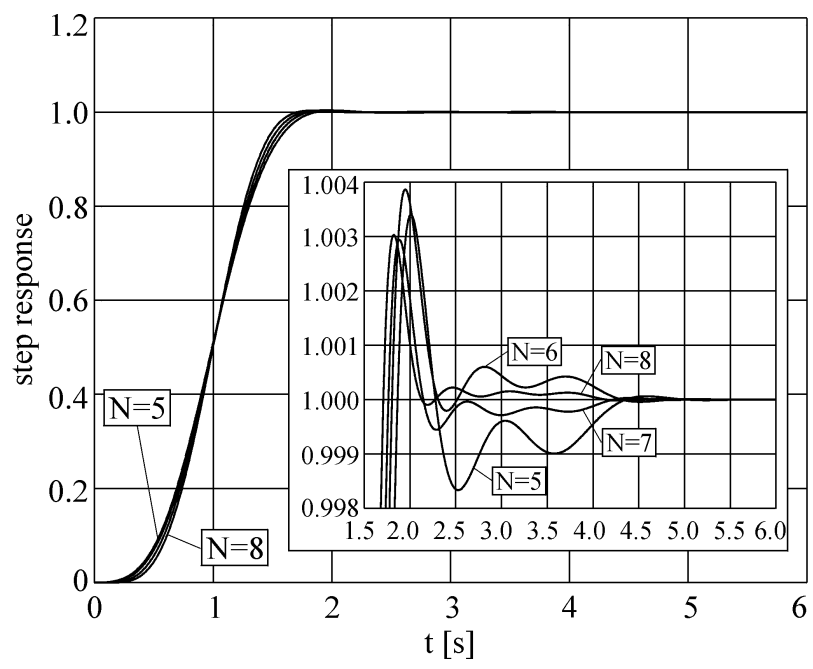

Figure 3. Step response of the optimum systems based on the fourth order moment, normalized to $t_{m}=1$.

approximation of constant. The bandwidth of quasiconstant group delay is extending well beyond cutoff frequency $\omega_{3 \mathrm{~dB}}$.

\subsection{Properties of the optimum systems}

The time-bandwidth product $\mathrm{P}_{\mathrm{n}}$, for various moments $\mathrm{n}$, and system orders $\mathrm{N}$, are shown in Figure 6. The curves in the diagram are given for all-pole systems presented in [9] together with the new results obtained with one pair of complex zeros. The products asymptotically converge to

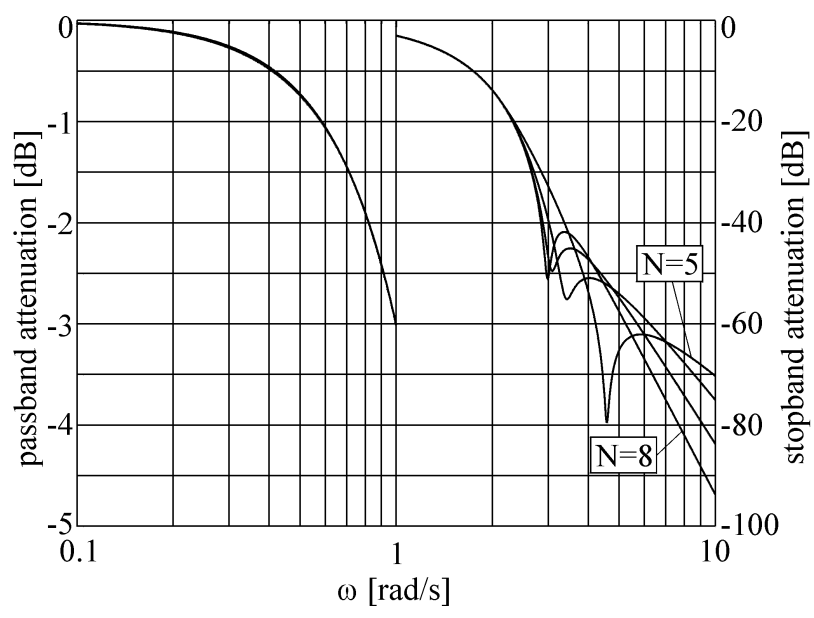

Figure 4. Amplitude response of the optimum systems based on the fourth order moment, normalized to $\omega_{3 \mathrm{~dB}}=1$.

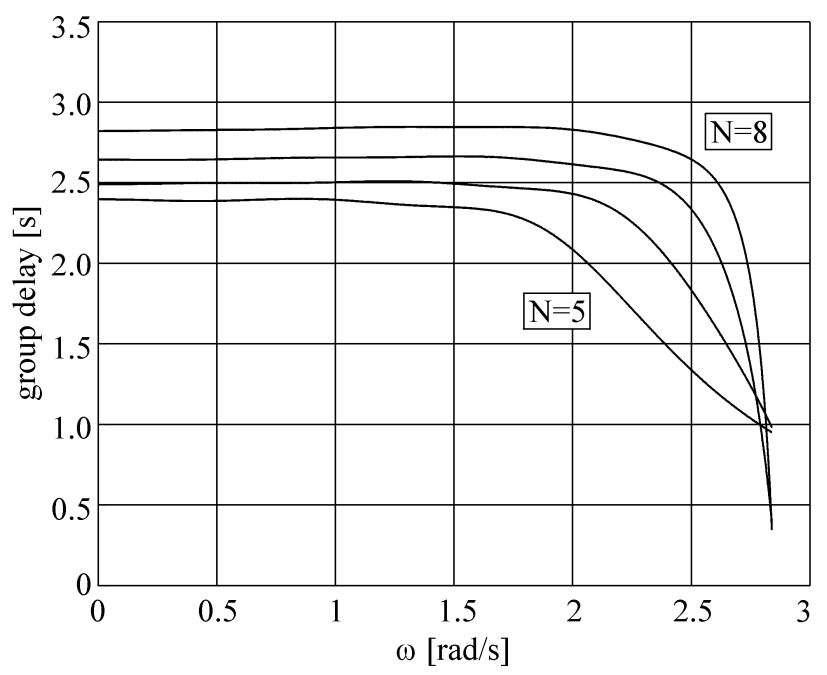

Figure 5. Group delay of the optimum systems based on the fourth order moment, normalized to $\omega_{3 \mathrm{~dB}}=1$.

the steady values for large $\mathrm{N}$. For the second moment it is well known limit $\mathrm{P}_{2}=1 / 2$, (4), while the other asymptotic values apparently depend on used moment order. The products approach to the values $0.83,1.14$ and 1.45 for $\mathrm{n}=4,6$ and 8 respectively. It is obvious that timebandwidth products are practically constant for systems with order $\mathrm{N} \geq 7$. The presence of zeros reduces the timebandwidth product. However, the contribution of the second pair of zeros is negligible, as it is visible in the extended diagrams in Figure 6. The effect of zeros is mainly in the reduction of the impulse response ringing, 

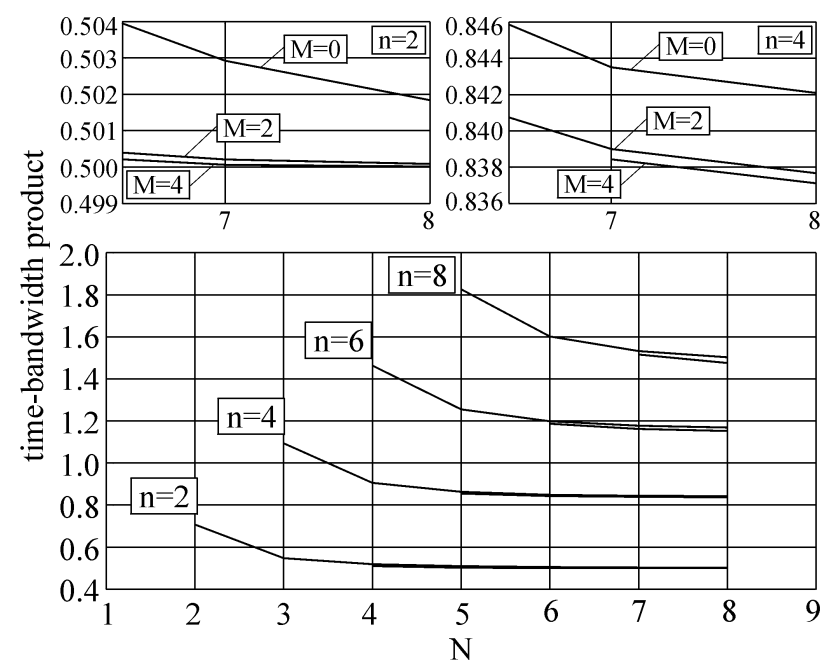

Figure 6. Time-bandwidth products of the optimum systems based on various moment orders.

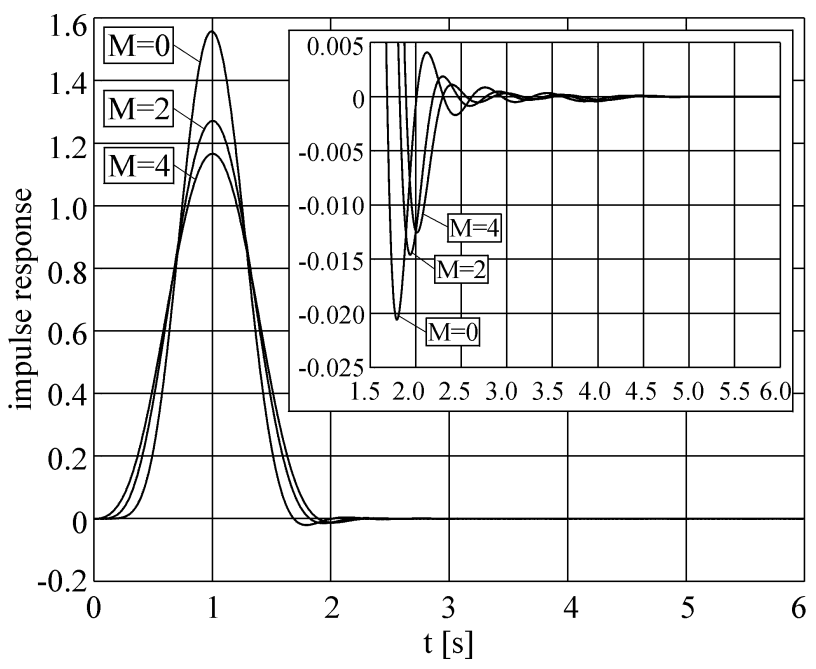

Figure 7. Impulse response of the optimum systems for various numbers of zeros, $N=8, n=4, t_{m}=1$.

as shown in Figure 7, but even there, the contribution of the second pair is negligible. This is the reason why we found sufficient to give here the numeric data for systems with one pair of zeros only. We also found that the finite real zeros can not improve the time-bandwidth product. Namely, the optimization procedure gives their position very far from the origin, i. e. far from the rest of poles and complex zeros.

The optimization results for all moment orders are similar in character to the systems of fourth moment order described above. For systems with second moment, the

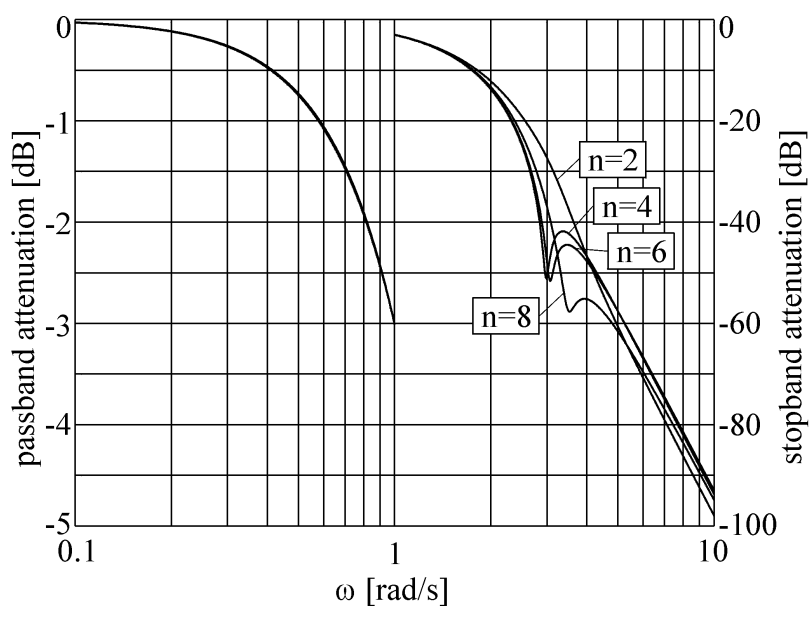

Figure 8. Amplitude response of the optimum systems based on various moment orders, $\mathrm{N}=8, \omega_{3 \mathrm{~dB}}=1$.

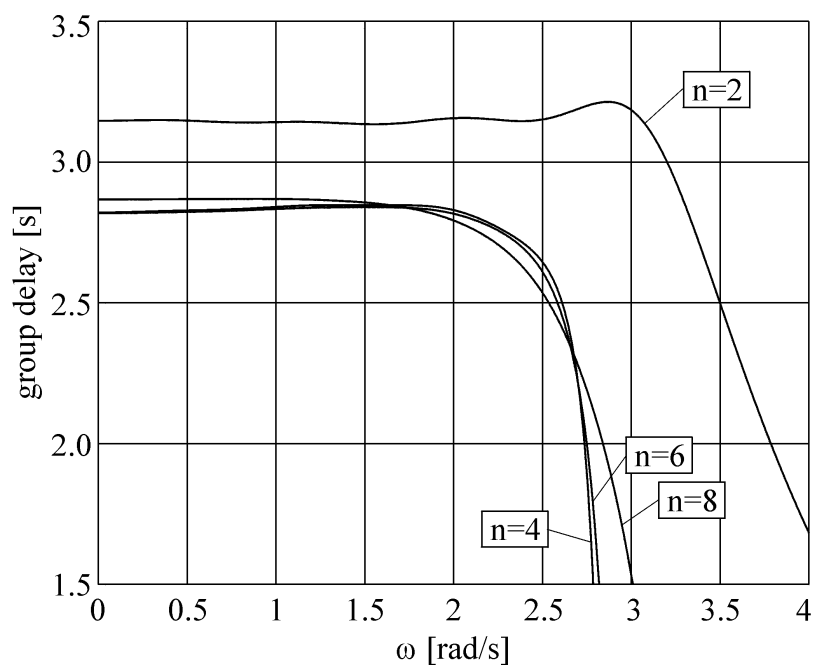

Figure 9. Group delay of the optimum systems based on various moment orders, $\mathrm{N}=8, \omega_{3 \mathrm{~dB}}=1$.

zeros are placed in the right half-plane, while for the other moment orders the zeros are in vicinity of the imaginary axis, Figure 1.

Impulse responses show shorter ringing for the higher moments, n, as it is expected from the used criteria (2). The higher moments with weighting function of higher power, in fact, more "punish" the "tails" of the responses. The impulse responses are Gaussian and quite symmetric. Undershoots for all responses are smaller than $1.5 \%$ and the overshoot of the step response is smaller than $0.6 \%$ for $\mathrm{n}=2,4,6$ and 8 . 
Amplitude attenuation in stop band is similar for all moment orders, as shown in Figure 8. In general, the slope in the transitional band is steeper for higher moment orders. The group delays approximate constants as shown in Figure 9.

\section{Conclusion}

By the numerical optimization of the time-bandwidth product based on the higher order moments, a new class of finite order systems has been obtained. Here we considered the influence of zeros, which we found useful for improvement of the ringing size and duration. We have shown that only one pair of complex zeros is practically interesting. The obtained systems have the largest energy concentration in time for a given bandwidth. The impulse response and step responses have small and short ringing.

\section{Acknowledgements}

This study was made at the Department of Electronic Systems and Information Processing of the Faculty of Electrical Engineering and Computing, University of Zagreb, Croatia. It was supported by Ministry of Science and Technology of Croatia, under grants No. 036024 and No. 036124.

\section{References}

[1] Chalk, J. H. H., "The Optimum Pulse Shape for Pulse Communication", Proceedings of IEE, Part 3, Vol. 97, 1950, pages 88-92.

[2] Papoulis A, Signal analysis, McGraw-Hill Inc., 1977.

[3] Kuchenbecker H. P., "Uber kausale Funktionen mit minimalem Zeit-Bandbreite-Produkt", Archiv der Elektrichen Ubertragung. H. 9, 1972, pages 259-264.

[4] Vucic M., Babic H., Filter Families with Minimum Impulse Response Moments, Proceedings of ISCAS 2000 - IEEE Symposium on Circuits and Systems, Geneva, Switzerland, May 28 - May 31, 2000, pages 557-560.

[5] Babic H., Vucic M., Filter Families with Minimum TimeBandwidth Products, accepted for presentation on ISCAS 2001 - IEEE Symposium on Circuits and Systems, Sydney, Australia, May 6-9, 2001.

[6] Fletcher R., Practical Methods of Optimization, Volume 1, John Wiley \& Sons, 1980.

[7] Kearfott R. B., Rigorous global search: Continuous Problems, Kluwer Academic Publishers, 1996.

[8] Zverev A. I., Handbook of Filter Synthesis, John Wiley \& Sons, 1967.

[9] Babic H., Vucic M., Systems with Minimum TimeBandwidth Product Based on the Higher Order Moments, Proceedings of the IASTED International Conference, Signal and Image Processing, Las Vegas, Nevada, USA, November 19-23, 2000, pages 231-234 